\title{
PROPER MOTIONS IN THE FAINT SKY VARIABILITY SURVEY
}

\author{
E. J. M. van den Besselaar ${ }^{1}$ and P. J. Groot ${ }^{1}$
}

The Faint Sky Variability Survey (FSVS) is aimed at finding photometrically and astrometrically variable objects. The main goal is to get a better understanding of binary evolution. This survey consists of 78 fields, which covers a total area of $21 \mathrm{deg}^{2}$ in the sky and it is sensitive for objects in the magnitude range of $17<\mathrm{V}<24$. (For a description see Groot et al. 2003)

The survey is done with the Wide Field Camera at the $2.5 \mathrm{~m}$ INT on La Palma. Every field is observed several times in the $\mathrm{V}$ band with an integration time of 10 minutes. The time interval between two images varies between 10 minutes and 3 years. To provide color information, every field is also observed once in the $\mathrm{B}$ and $\mathrm{I}$ bards. The fields are chosen at midto high-galactic latitudes to prevent crowding and to probe the galactic halo as well as the galactic disk. Furthermore, the fields are observed at zenith distances of $z<30^{\circ}$. The fields are also devoid of bright stars $(\mathrm{V}<10)$ to prevent charge overflows and diffraction patterns.

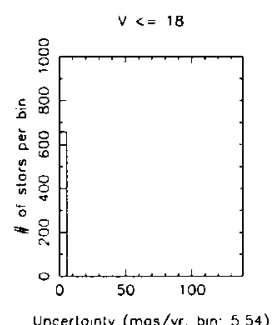

$20<v<2=21$

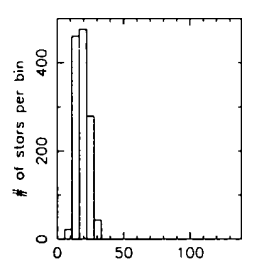

uncerionty (mos/yr, bin' 554$)$
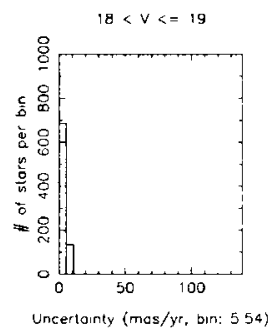

$21<v<-22$

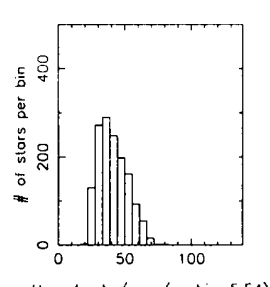

uncerloniny (mos/yr, bin: 5.54 )
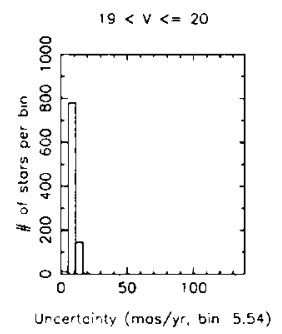

$v>22$

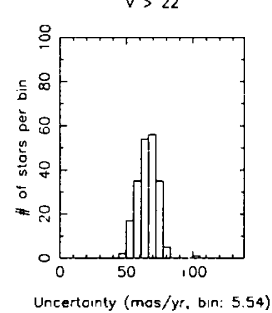

Fig. 1. A histogram of the uncertainties of the derived proper motion given in mas/yr as a function of magnitude range. Fainter objects have larger uncertainties, because the central position of fainter objects is more difficult to derive.

\footnotetext{
${ }^{1}$ Department of Astrophysics, University of Nijmegen, P.O. Box 9010, 6500 GL Nijmegen, The Netherlands (besselaar@astro.kun.nl, pgroot@astro.kun.nl).
}
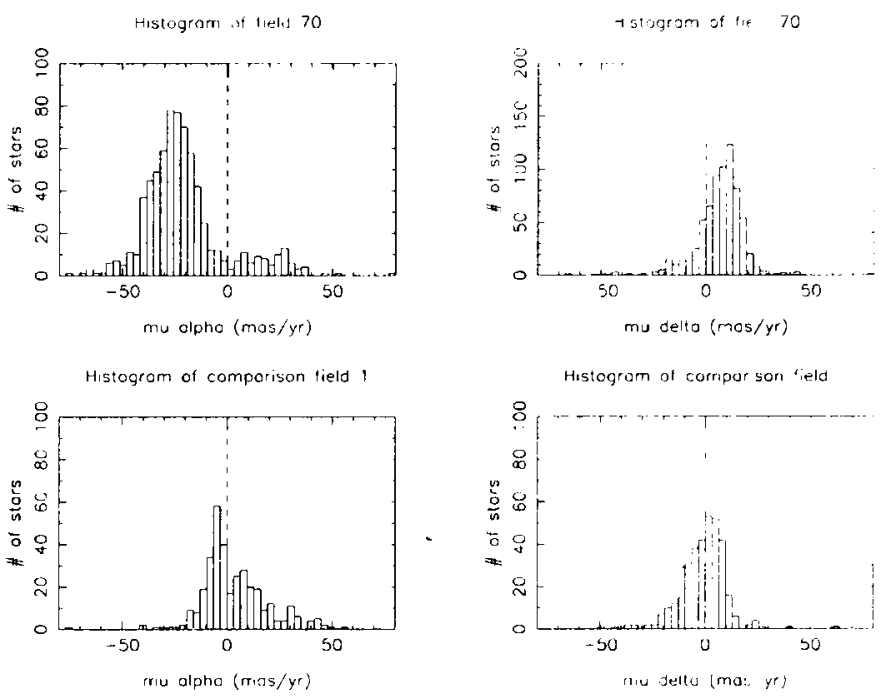

Fig. 2. A histogram of the proper motions in the right ascension and declination. In the upper field, a group of objects is moving at the same speed and in the same direction, while the iower field, a comparison field, does not show this behaviour.

We are measuring the proper motions of the stars in this survey. To do this, we have to perform a coordinate-transformation to align the coordinates of the input and reference image. This is done with the IRAF package DIFIMPHOT. After the transformation, the images are cut in smaller pieces around the objects. A 2D Gaussian fit is performed on these smaller images. From the central positions derived from this fit, the proper motion of the objects is determined. The uncertainty in the proper motion is dependent of the magnitude of the objects. This can be seen in Fig. 1. A color-color diagram is produced and we are interested in objects outside the mainsequence, in particular in white dwarf - red dwarf binary candidates. Spectra will be taken of these candidates to classify the objects.

This method has so far been applied to a small number of fields. One of these fields seems to be very interesting. A group of objects is moring with the same speed and in the same direction (Fig. 2). The analysis of this excess is ongoing in the complete effort of deriving the proper motion of the objects in the Faint Sky Variability Survey.

\section{REFERENCES}

Groot, P. J., et al. 2003, MNRAS, 339, 4:2 